\title{
Study on the Existing Destruction Methods for Abandoned Explosion Tear Gas of CAPF
}

\author{
Fu Peng, Feng Lan \\ Training Department, Engineering University of CAPF, Xi'an, 710086, P.R.China
}

Key words: explosion tear gas; destruction methods; Incineration / blasting, dissolution;

\begin{abstract}
In this paper, based on the characteristics and actual demand of the safety management to weapons and equipment, techniques and methods of destruction of anti-riot ammunition used by CAPF were discussed. Combing the main structure characteristics and function of the common anti-riot ammunition, some conventional destruction methods were put into use for anti-riot ammunition destroyed using through theoretical analysis and experimental verification methods. The study support important significance to maintenance equipment safety and improve combat effectiveness.
\end{abstract}

\section{Introduction}

Antiriot ammunition including tear gas, knock bombs and other special bombs were well known in modern counter-terrorism operations as the essential weapons [1], but the attendant abolition of Antiriot ammunition has become more and more prominent. On the one hand, the average storage life of anti-riot ammunition was only five years [2], which will make the disposal work of obsolete ammunition become very hard in such a short time; on the other hand, anti-riot ammunition is accompanied by the progress and development of the non-lethal technology, sorts of ammunition retired and update faster and faster, which also increase the difficulty of the disposal work. Because the structure of riot ammunition was widely different from the conventional munitions, it determined that the technology and methods of disposal processing was also different. The primarily aims of disposal work were safe handling two types of abandoned anti-riot ammunition timely through the relevant technical measures, including some expired because of exceeding the storage period or existing security risks, some retired because of the equipment updated. Eliminating security risks not only ensure staff and workplace safety but also encourage the smooth progress of scientific research equipment.

\section{The characteristics of disposal work for obsolete ammunition}

\section{Waste object}

Riot ammunition is a kind of non-lethal weapons, which usually achieve to operational effectiveness by releasing sound, light and smoke, fog, aerosols and other irritants as a fire or explosion, not by strike, damage target generally. For this reason, the killing capacity for staff and object was limited, and the destruction that the riot ammunition damage effect may be caused is less than conventional ammunition by an accident. For example, there was only a small amount black powder inside of the explosive tear gas, which limited the detonation capacity and result in the poor sympathatic detonation; combustion tear gas usually load a small amount of propellant inside, whose main ingredient is potassium chlorate, combustion agent and a small amount of metal particles. The mode of action was often burning, and it is difficult to reach the condition of produce detonation. In this paper, the most widely used explosion tear gas was studied as a riot ammunition bomb with typical feature [3], and its structure shown in Fig.1. 


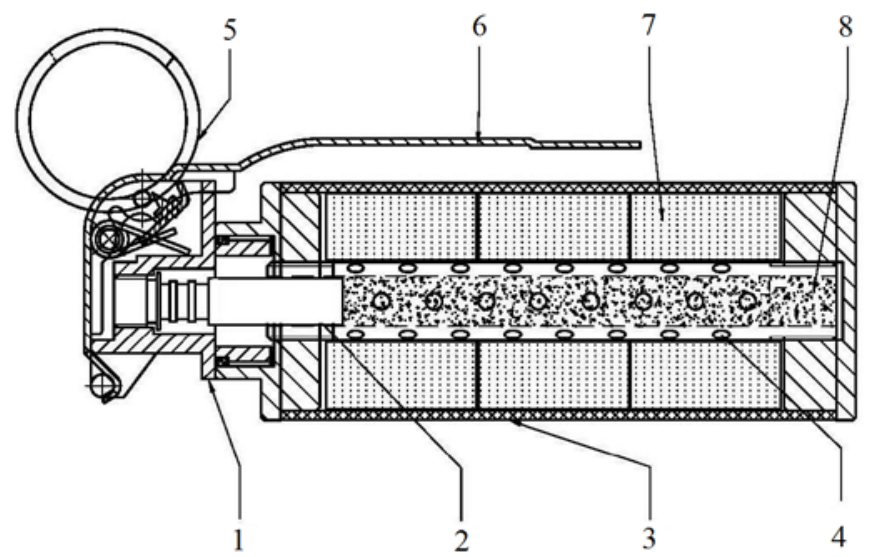

1-connection receptacle; 2-percussion mechanism; 3-projectile body;

4-delayer; 5-starting mix; 6-spur guard; 7-charge; 8-firing pin;

Fig.1: Construction of explosion tear-gas

The action principle was shown as following steps: When explosion tear gas was thrown by hand, the propellant inside can causes the irritants CS (o-chloro-benzylidene malononitrile) sublimation by burning, and the aerosols in the air formed and began to chase the crowd. Muzzle velocity is relatively small because of the indirect contact with the target, so the projectile material usually adopts paper that can satisfy for the requirement of strength. Internal charge press-fit using two grain filling, because it contains more functional agent, the shelf life is much smaller than conventional propellant, such that the overall life expectancy is short of ammunition. Between components using thread structure, for the sake of better sealing, thread after assembled directly bonded together, so they can not be molded by hand once the demolition. Metal projectile and ignition devices typically have recycling value, and try to taken protective measures when handling in order to re-use it.

\section{Requirements}

As an important part of operational equipment support, disposal work for obsolete anti-riot ammunition was also put forward higher requirements with the continuous improvement of the overall force protection capabilities. In general, technologies of disposal work for waste anti-riot ammunition should reference the latest research results for the abolition of conventional ammunition, mainly reflected in the following aspects:

(1) Study on new ammunition destruction modes including water jet cutting, incinerator and biological composting, wherein the abrasive water jet technology was generally acknowledged to have good prospects in terms of anti-riot ammunition disposal.

(2) Study on contamination control in the ammunition disposal process, such as prohibiting or restricting the large equivalent burning or blasting [4], and develop the biodegradation, adsorption, membrane treatment methods for the waste water and smoke of destruction processing.

(3) Study on automating and intelligent of disposal work for waste anti-riot ammunition, it not only effectively guaranteed the safety of the operator, but also help improve the technological level and destruction efficiency.

Based on the structural features of anti-riot ammunition, the process security should be ensured at three areas including the equipment, technology and operation. Although sensitivity of the main charge is less than high explosive, severe impact and friction is prohibited during operation. In order to prevent accidents, make sure that there is no explosive hazard nearby and as the case taken down the sensitivity by a sense of other technical measures. 


\section{Introduction of destruction methods}

\section{Selection}

Ensuring the process safety is the premise and foundation of disposal work for anti-riot ammunition. Firstly, classify management should be ascertained in accordance with composition, ignition, amount and types of ammunition. High-risk or unknown-risk ammunition should be treated separately, and the same batch of ammunition should be processed simultaneously. Secondly, whether anti-riot ammunition could be demounted or not was in accordance with the structural characteristics before choosing the most timely destruction method, shown in Fig.2.

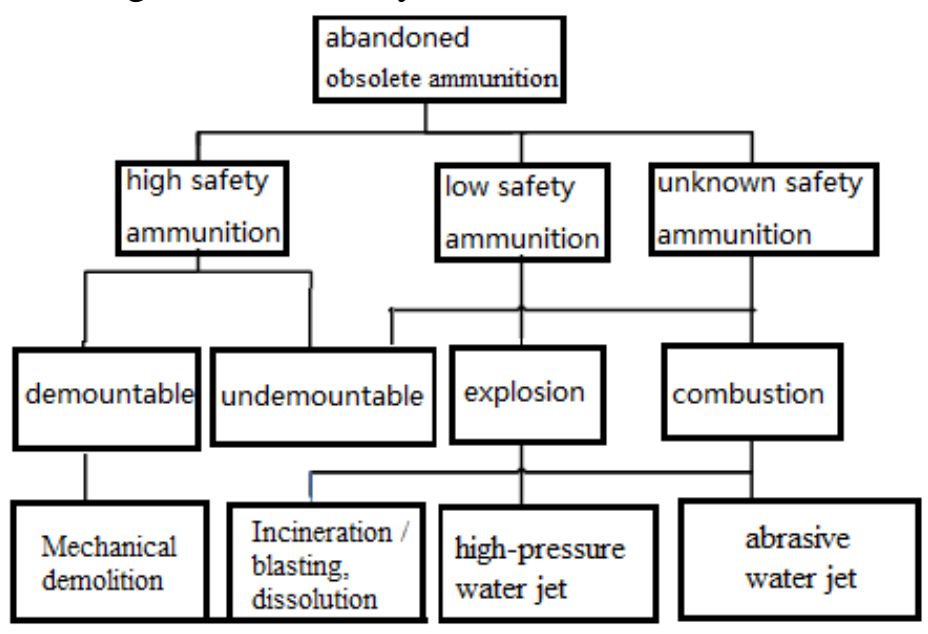

Fig.2: Classification of disposal work for obsolete ammunition

Currently, the routine department mode of waste anti-riot ammunition has been formed to take advantage of decomposition after disassembly, and supplemented by controlled burning and blasting processing [5].

\section{Mechanical demolition}

Mechanical demolition and then pouring out the charge is regarded as the most commonly used method, and it can extremely recover metals materials and charge, but there is the higher security requirement in the process. The charge of riot ammunition was complex and diverse, so its demolition should be flexibility in the pursuit of efficiency and emphasis on safety. After the demolition, the main components is first recovered, and other smaller or valuable component for can be recovery used for the further processing. CAPF Engineering University has developed a kind of dismantling equipment for combustion tear gas, explosion tear gas and electric primer riot bomb, shown in Fig.3. The device used a steel tool for damnation of projectile body and firing mechanism. In order to prevent the unexpected ignition of firing mechanism by the mechanical and thermal effects in the process, researchers designed a coolant spray device to prevent sparks and excessive temperature changes. The device is widely adaptable, but limited by the destruction efficiency, only suitable for small batch processing. 

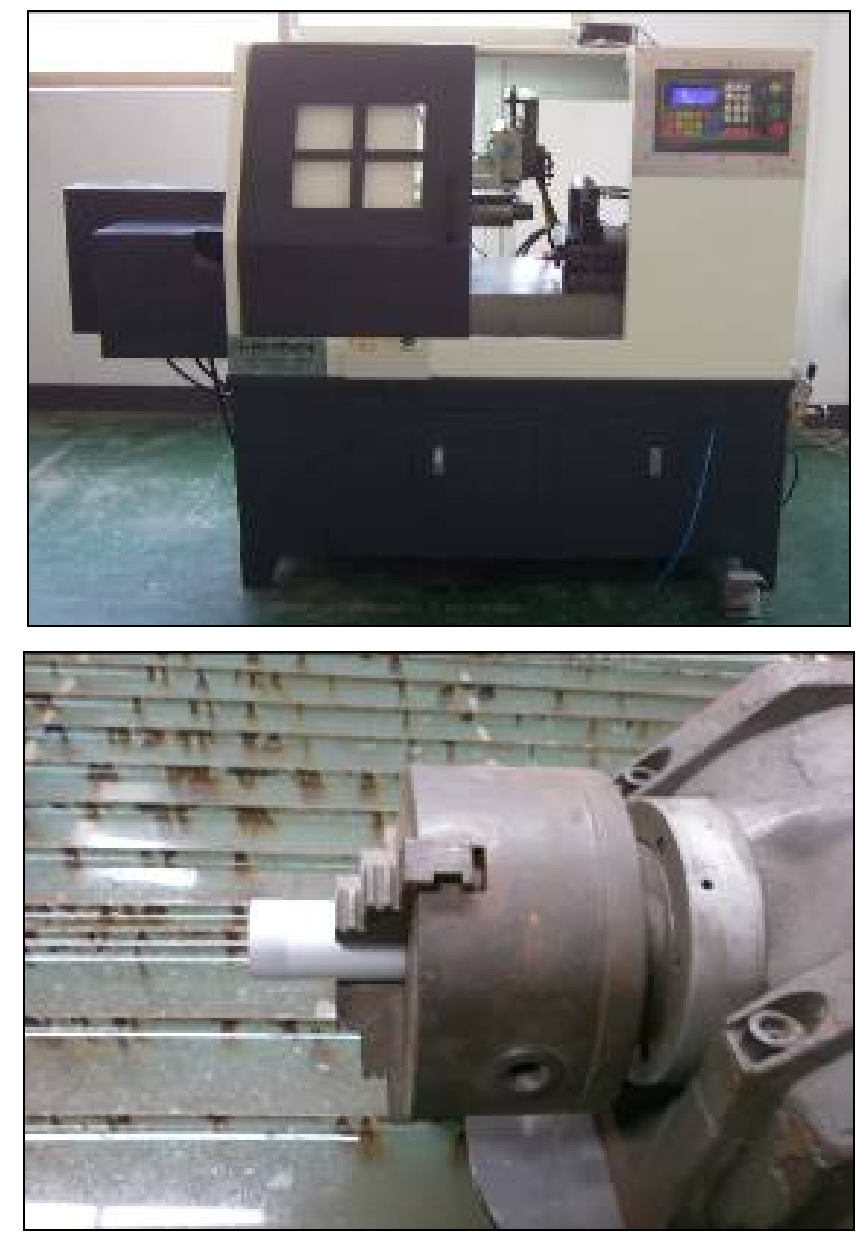

Fig.3: Mechanical demolition device

\section{Incineration / blasting, dissolution method}

Now, incineration, explosion and dissolution method focused in shells or full charge of some anti-riot ammunition with more dangerous, or difficult to disassemble or low re-use value. It can be divided that incineration method is mainly used in the non-explosive species, such as various types of smoke bombs, combustion tear gas and so on. The method often rely on adding fuel to burn, so it is simple, reliable, economical and thorough, while the burning site and weather conditions limited its scope.

Explosion method was thought to adapt to all types of explosive species, such as knock bombs, explosive tear gas and so on. Its destruction operations generally located in the special destroy venues with rugged bunkers, made use of non-electric blasting detonator network Road to detonate TNT explosives or other civilian with the mode of segments and once. Explosion method had well adaptability, especially adapt to the large volume or larger explosion hazards, but it seriously demands on the environment and its destruction costs are very high. When a larger number destruction is needed, more remote areas must be selected to implement accordance to batches, job points and fractionation.

Dissolution method focused on the destruction operations of main charge, and usually directly made use of soaked with water. Because per chlorate in the main charge can dissolve in water, can also use chemical solvents to dissolve under special circumstances. The method is simple, feasible and low environmental requirements, but may cause environmental pollution if it was improper used. For example, Rs97-2 combustion tear gas owns the ternary grain components (aluminum/ potassium chlorate/ sugar), can be dissolved by use of sodium hydroxide solution.

$$
\mathrm{KClO}_{3}+2 \mathrm{Al}+2 \mathrm{NaOH} \rightarrow 2 \mathrm{NaAlO}_{2}+\mathrm{H}_{2} \mathrm{O}+\mathrm{KCl}
$$

(a) 


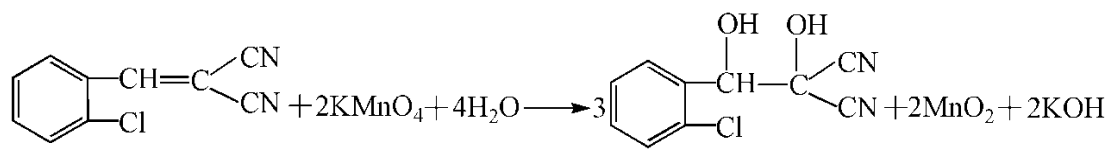

(b)

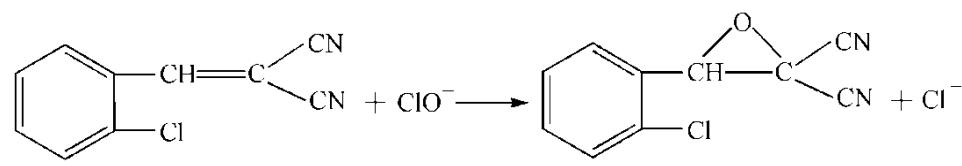

(c)

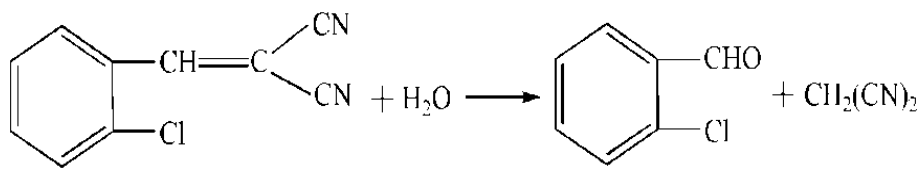

(d)

Known by the chemical reaction equation, main products were potassium chloride and sodium aluminates. Both of them belong to toxic substances, less harmful to the environment, so it can be directly discharged, as shown in Fig.4.
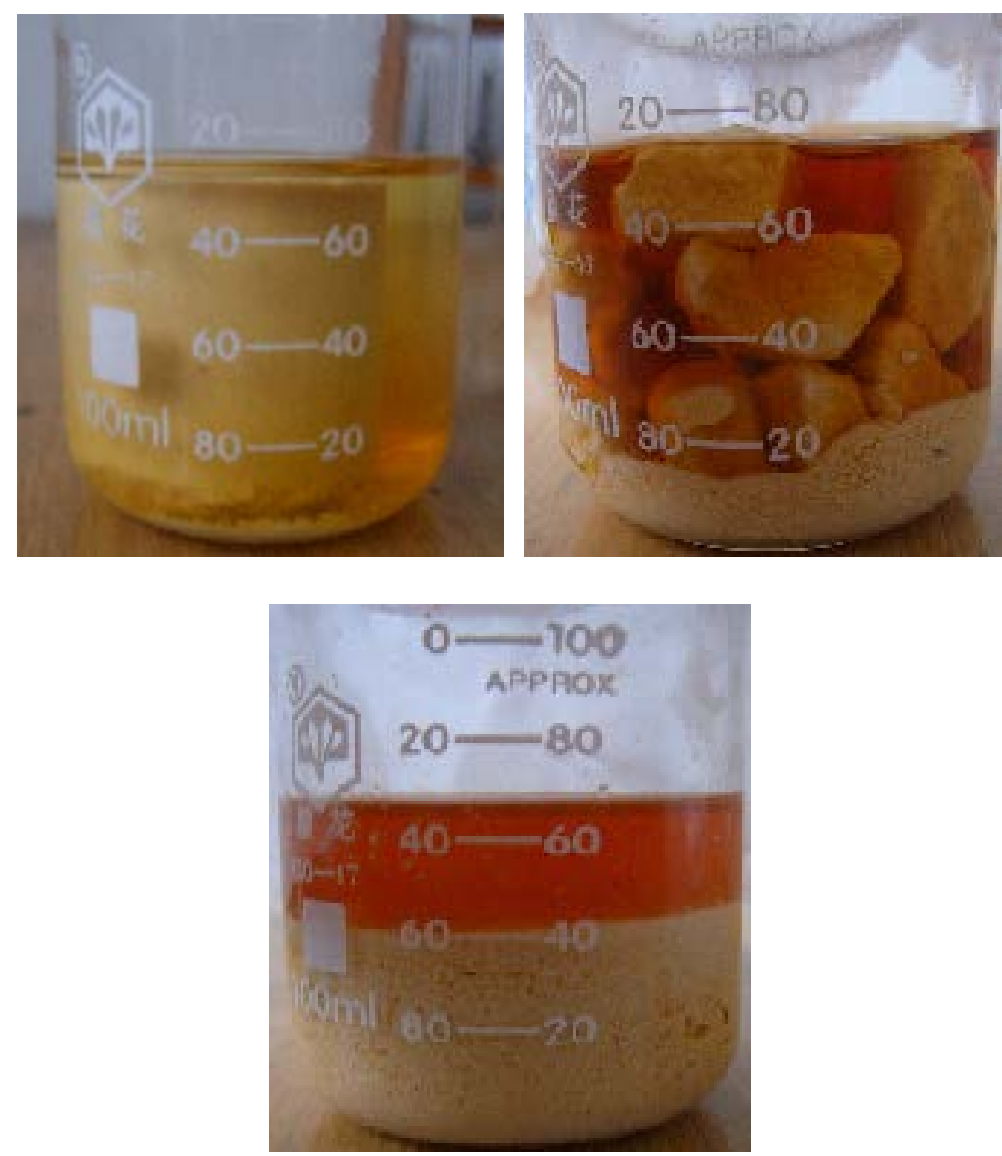

Fig.4 Dissolution method for components

\section{Conclusions}

Researching the destruction theory and technology for anti-riot ammunition can eliminate adverse factors about the developing of waste work, thus improved and promoted the overall capacity to their safe and effective management of anti-riot ammunition. two key issues were drawn as the following conclusions: 
1) Disposal work for obsolete anti-riot ammunition must be based on the internal structure and mode of action of anti-riot ammunition, must ensure the process safety as a precondition.

2) Burning / blasting, dissolved destruction method must timely consider the treatment of incidental smoke and waste water generated in the process that might affect the environment and people.

\section{Acknowledgement}

In this paper, the research was sponsored by the Nature Science Foundation of Shaanxi Province (Project No. 2014JM7284) and the Post-doctoral Science Foundation of China (Grant No. 2012M521945).

\section{Reference}

[1] Zhu Shuang. High pressure water jet cutting technology and application development [C], 50th anniversary Changsha Mining and Research Institute Proceedings, 2006, 10: 87-88.

[2] Xue Shengxiong. Pressure water jet technology engineering [M] Hefei: Hefei University of Technology, 2006.

[3] Gu Cheng Chu and He Yunhua. The analysis and research on cutting mechanism technology high-pressure water jet and cutting technology of abrasive water jet [J], mining machinery, 2004, 3: 48-52.

[4] Zhong Shuliang. Study on water jet cutting technology for explosives [D] Beijing: Chinese Academy of Engineering Physics, Doctoral Dissertation, 2006.

[5] Li Jinghai. Study on Explosives Pyrotechnics in processing and recycling of abandoned missile [J], defense technology base, 2007, 6 (6): 49-52. 